\title{
Research of Dracocephalum palmatum S. and Dracocephalum ruyschiana L. originating from Yakutia and identification of metabolites by tandem mass spectrometry
}

\author{
Mayya Razgonova ${ }^{1,3,}{ }^{*}$, Zhanna Okhlopkona ${ }^{2}$, and Kirill Golokhvast ${ }^{1,3,4,5}$ \\ ${ }^{1}$ N.I. Vavilov All-Russian Institute of Plant Genetic Resources, 190000, 42-44, B. Morskaya, Saint- \\ Petersburg, Russian Federation \\ ${ }^{2}$ Department of Biology, North-Eastern Federal University, 677000, 58, Belinsky str., Yakutsk, \\ Russian Federation \\ ${ }^{3}$ Far Eastern Federal University, 690950, 8, Sukhanova, Vladivostok, Russian Federation \\ ${ }^{4}$ Pacific Geographical Institute, Far Eastern Branch of the Russian Academy of Sciences, 690041, 7 , \\ Radio, Vladivostok, Russian Federation \\ ${ }^{5}$ Siberian Federal Scientific Centre of Agrobiotechnology, 633501, Centralnaya, Presidium, \\ Krasnoobsk, Russian Federation
}

\begin{abstract}
Dracocephalum palmatum Stephan and Dracocephalum ruyschiana L. contains a large number of target analytes, which are biologically active compounds. High performance liquid chromatography (HPLC) in combination with a BRUKER DALTONIKS ion trap (tandem mass spectrometry) was used to identify target analytes in extracts of $D$. palmatum Stephan and D. ruyschiana L., originating from Yakutia. The results of initial studies revealed the presence of 61 compounds, of which 53 were identified for the first time in genus Dracocephalum. These are flavones: Apigenin 8-C-pentoside-6-C-hexoside, Apigenin 7-sulfate; Chrysin 6-C-glucoside, Chrysin glucuronide; flavanols: Kaempferol, Dihydrokaempferol, Astragalin; flavan-3-ol (epi)Catechin, phenolic acids: Methylgallic acid; Hydroxy methoxy dimethylbenzoic acid; Ellagic acid; Caffeoylshikimic acid; Prolithospermic acid; 3,4-O-dicaffeoylquinic acid; salvianolic acid G; stilbenes pinosylvin and resveratrol; anthocyanins Petunidin, Pelargonidin-3-O-glucoside; Peonidin-3-O-glucoside; Cyanidin 3-(acetyl)hexose; perillic acid; lignans: Hinokinin, Dimethylsecoisolariciresinol, Podophyllotoxin, carotenoids: Apocarotenal, 5,8epoxy-alpha-carotene; etc.
\end{abstract}

\section{Introduction}

Yakutia is a subject of the Russian Federation, which is one of the largest subnational subjects. Yakutia is located in several climatic zones and on its territory, there is an amazing variety of plant resources, including medicinal plants. One of these medicinal plants are plants belonging to the genus Dracocephalum. Dracocephalum palmatum

\footnotetext{
*Corresponding author: m.razgonova@vir.nw.ru
} 
Stephan and Dracocephalum ruyschiana L. (Section Buguldea Benth., Subgenus Eudracocephalum Briq., Genus Dracocephalum L., Family Lamiaceae), are a perennial rhizomatous plant with numerous stems and ovate-rounded, pinnatifid leaves, and purple flowers on short stalks gathered in false whorls at the end of the stems in an oblong inflorescence (Fig. 1).

Several plants belonging to the genus Dracocephalum have been reported to possess anticancer, antioxidant, and cardio-protective effects [32]. Lee et al. (2020) reported about the anticancer activity of $D$. palmatum Stephan, dried leaves of D. palmatum and the effects of various extracts on intracellular reactive oxygen species (ROS) levels, cell cycle distribution, apoptotic cell population and apoptosis-related protein levels were extensively studied to assess potential anti-cancer activity. Research by Kim et al. (2020) was aimed at evaluating the tumor suppressive effect of $D$. palmatum extract in diffuse large cell lymphoma and the underlying mechanism. Fractionation of D. palmatum extract by thin layer chromatography and analysis of the fraction with biologically active compounds based on liquid chromatography/mass spectrometry demonstrated that flavonoids may be responsible for most, if not all, of the anti-lymphoma effect. Extensive research is currently underway to identify bioactive flavonoids from $D$. palmatum extracts.

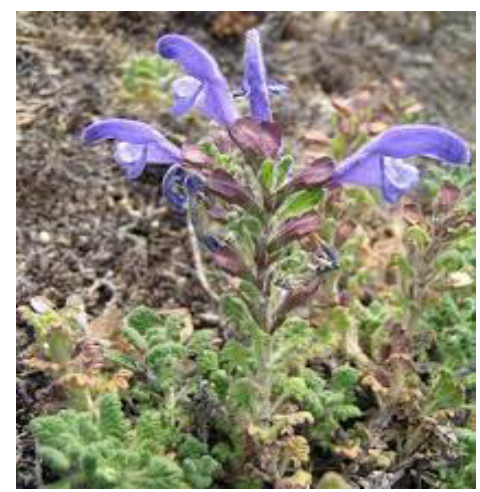

A

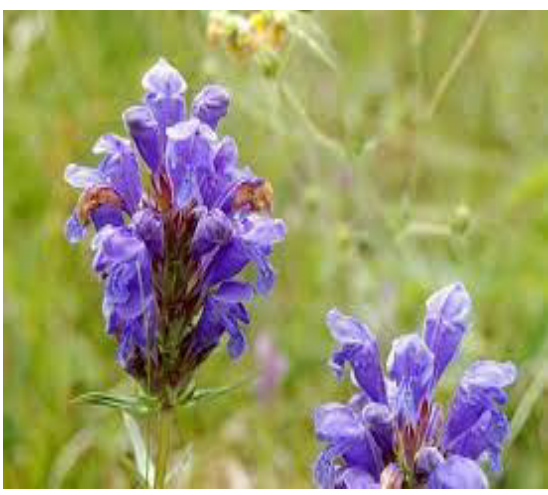

B

Fig. 1. A: Dracocephalum palmatum S. B: Dracocephalum ruyschiana L.

This research examines a detailed metabolomic and comparative analysis of leaves and twigs of D. palmatum Stephan and D. ruyschiana L., originated from Yakutia and collected by scientists of North-Eastern Federal University.

\section{Methods}

\subsection{Materials}

As an object of research, we used leaves and twigs of D. palmatum Stephan and $D$. ruyschiana L., originated from Yakutia. All samples were morphologically authenticated according to the current standard of Russian Pharmacopoeia [29].

\subsection{Chemicals and Reagents}

HPLC-grade acetonitrile was purchased from Fisher Scientific (Southborough, UK), MSgrade formic acid was from Sigma-Aldrich (Steinheim, Germany). Ultra-pure water was 
prepared from a SIEMENS ULTRA clear (SIEMENS water technologies, Germany), and all other chemicals were analytical grade.

\subsection{Fractional maceration}

To obtain highly concentrated extracts, fractional maceration was applied. In this case, the total amount of the extractant (methyl alcohol of reagent grade) is divided into 3 parts and is consistently infused on Dracocephalum with the first part, then with the second and third. The infusion time of each part of the extractant was 7 days.

\subsection{Liquid chromatography}

HPLC was performed using Shimadzu LC-20 Prominence HPLC (Shimadzu, Japan) was used, equipped with an UV-sensor and a Shodex ODP-40 4E reverse phase column to perform the separation of multicomponent mixtures. The gradient elution program was as follows: $0.01-5 \mathrm{~min}, 100 \% \mathrm{CH}_{3} \mathrm{CN}$; 5-45 $\mathrm{min}, 100-25 \% \mathrm{CH}_{3} \mathrm{CN}$; $45-55 \mathrm{~min}, 25-0 \%$ $\mathrm{CH}_{3} \mathrm{CN}$; control washing 55-60 min $0 \% \mathrm{CH}_{3} \mathrm{CN}$. The entire HPLC analysis was done with a ESI detector at wavelengths of $230 \mathrm{\eta m}$ and $330 \mathrm{\eta m}$; the temperature corresponded to $17^{\circ} \mathrm{C}$. The injection volume was $1 \mathrm{ml}$.

\subsection{Mass spectrometry}

MS analysis was performed on an ion trap amaZon SL (BRUKER DALTONIKS, Germany) equipped with an ESI source in negative ion mode. The optimized parameters were obtained as follows: ionization source temperature: $70^{\circ} \mathrm{C}$, gas flow: $4 \mathrm{l} / \mathrm{min}$, nebulizer gas (atomizer): $7.3 \mathrm{psi}$, capillary voltage: $4500 \mathrm{~V}$, end plate bend voltage: $1500 \mathrm{~V}$, fragmentary: $280 \mathrm{~V}$, collision energy: $60 \mathrm{eV}$. A four-stage ion separation mode (MS/MS mode) was implemented.

\section{Results}

Six of the most consumed extracts of D. palmatum Stephan and D. ruyschiana L. were analyzed by HPLS-MS/MS ion trap to better interpret the diversity of available phytochemicals. All of them have a rich bioactive composition. The structural identification of each compound was carried out on the basis of their accurate mass and MS/MS fragmentation by HPLC-ESI-ion trap-MS/MS. Sixty-one biologically active compound was successfully identified characterized by comparing fragmentation pattern and retention time. Other compounds were identified by comparing their MS/MS data with available literature. All the identified compounds along with molecular formulas, $\mathrm{m} / \mathrm{z}$ calculated and observed, MS/MS data, and their comparative profile for two varieties of Dracocephalum are summarized in Table 1. The total ion chromatograms of D. ruyschiana L. extracts in positive and negative ionization modes are presented in Figure 2, respectively. 


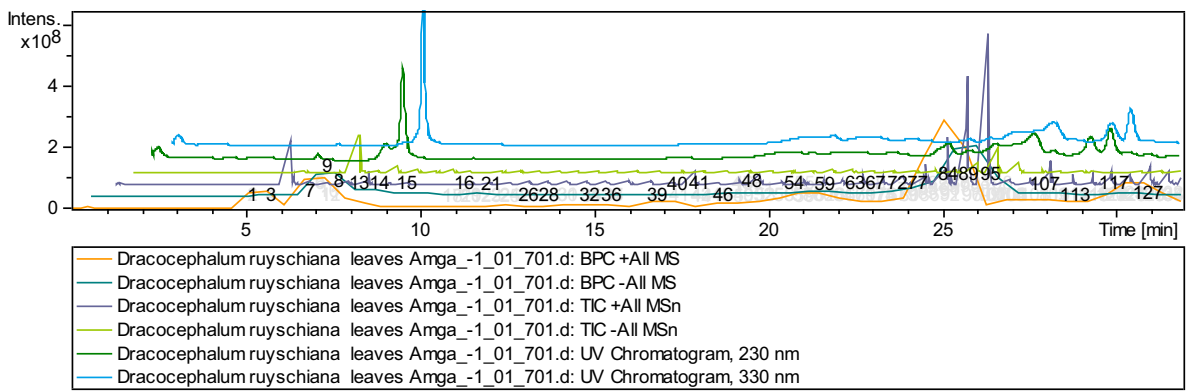

Fig. 2. Chemical profiles of $D$. ruyschiana $\mathrm{L}$. sample represented total ion chromatogram.

Table 1. Compounds identified from the extracts of D. palmatum Stephan and D. ruyschiana L. in positive and negative ionization modes by HPLC-ion trap-MS/MS.

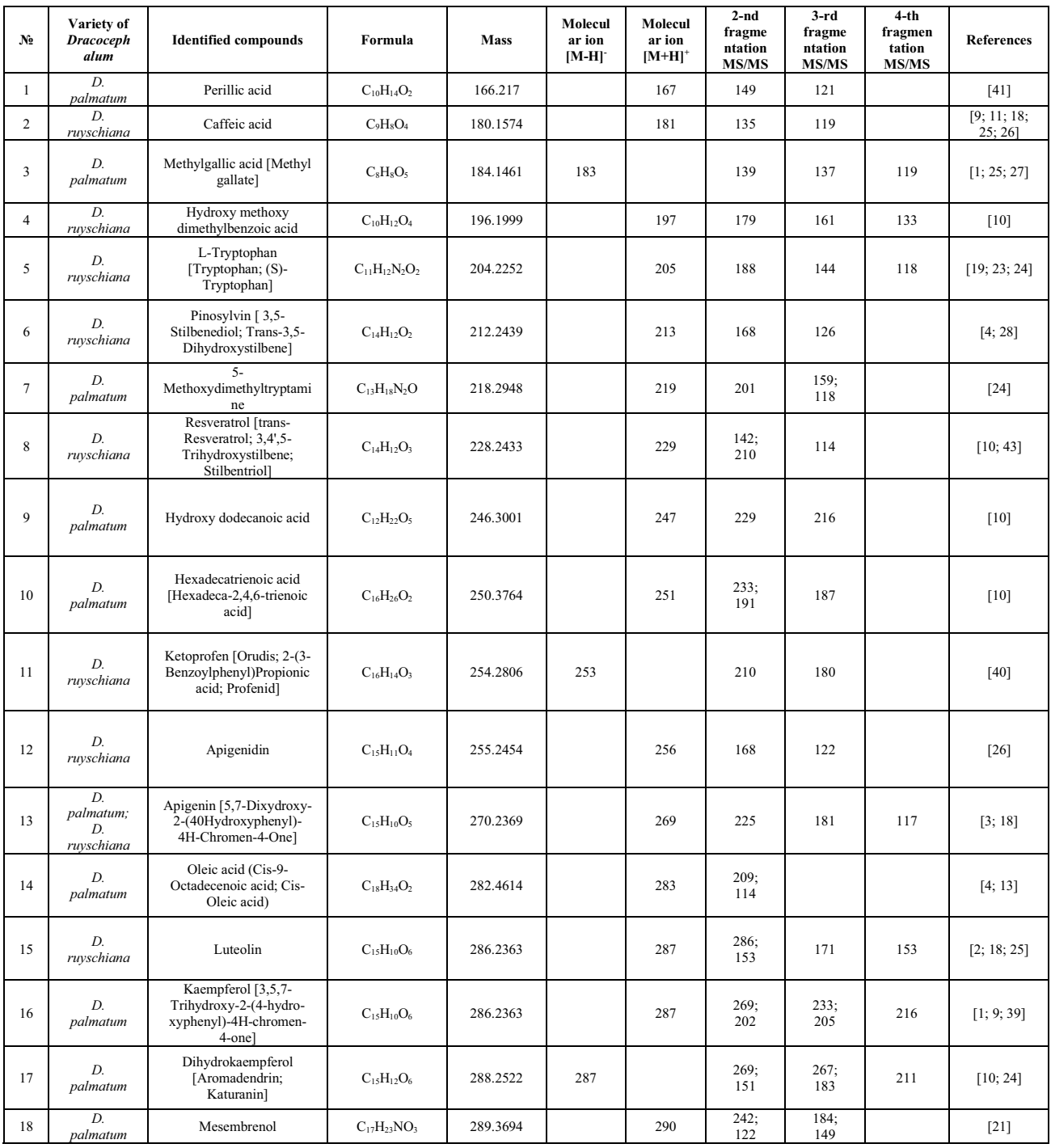




\begin{tabular}{|c|c|c|c|c|c|c|c|c|c|c|}
\hline 19 & $\begin{array}{c}D . \\
\text { ruyschiana }\end{array}$ & (epi)catechin & $\mathrm{C}_{15} \mathrm{H}_{14} \mathrm{O}_{6}$ & 290.2681 & & 291 & $\begin{array}{l}273 ; \\
117\end{array}$ & $\begin{array}{l}255 ; \\
145\end{array}$ & & $\begin{array}{c}{[9 ; 10 ; 24} \\
43]\end{array}$ \\
\hline 20 & $\begin{array}{c}D . \\
\text { ruyschiana }\end{array}$ & $\begin{array}{l}\text { Ellagic acid [Benzoaric } \\
\text { acid; Elagostasine; } \\
\text { Lagistase; Eleagic acid] }\end{array}$ & $\mathrm{C}_{14} \mathrm{H}_{6} \mathrm{O}_{8}$ & 302.1926 & 301 & & 284 & 221 & 112 & {$[1 ; 25 ; 30]$} \\
\hline 22 & $\begin{array}{c}D . \\
\text { palmatum }\end{array}$ & Petunidin & $\mathrm{C}_{16} \mathrm{H}_{13} \mathrm{O}_{7+}$ & 317.2702 & & 318 & $\begin{array}{l}166 ; \\
300 \\
\end{array}$ & 121 & & {$[10]$} \\
\hline 23 & $\begin{array}{l}D . \\
\text { ruyschiana }\end{array}$ & $\begin{array}{l}\text { 1-O-(4-Coumaroyl)- } \\
\text { glucose }\end{array}$ & $\mathrm{C}_{15} \mathrm{H}_{18} \mathrm{O}_{8}$ & 326.2986 & 325 & & 145 & 117 & & {$[9 ; 22]$} \\
\hline 25 & $\begin{array}{l}D . \\
\text { ruyschiana }\end{array}$ & Docosahexaenoic acid & $\mathrm{C}_{22} \mathrm{H}_{32} \mathrm{O}_{2}$ & 328.4883 & 327 & & $\begin{array}{l}309 ; \\
201\end{array}$ & $\begin{array}{c}291 \\
171\end{array}$ & 273 & [34] \\
\hline 26 & $\begin{array}{c}D \\
\text { ruyschiana }\end{array}$ & $\begin{array}{c}\text { Caffeoylshikimic acid [5- } \\
\text { O-Caffeoylshikimate] }\end{array}$ & $\mathrm{C}_{16} \mathrm{H}_{15} \mathrm{O}_{8}$ & 335.2855 & 335 & & 179 & 135 & 133 & {$[27 ; 31]$} \\
\hline 27 & $\begin{array}{c}\text { D. } \\
\text { palmatum }\end{array}$ & Salvianolic acid $\mathrm{G}$ & $\mathrm{C}_{18} \mathrm{H}_{12} \mathrm{O}_{7}$ & 340.2837 & & 341 & $\begin{array}{l}296 ; \\
208\end{array}$ & $\begin{array}{l}278 \\
208\end{array}$ & $235 ; 164$ & {$[11 ; 41]$} \\
\hline 28 & $\begin{array}{c}D . \\
\text { palmatum }\end{array}$ & $\begin{array}{l}\text { 1-Caffeoyl-beta-D- } \\
\text { glucose [Caffeic acid-3- } \\
\text { O-beta-D-glucoside] }\end{array}$ & $\mathrm{C}_{15} \mathrm{H}_{18} \mathrm{O}_{9}$ & 342.298 & 341 & & $\begin{array}{l}178 \\
119\end{array}$ & 135 & & {$[9 ; 19]$} \\
\hline 29 & $\begin{array}{c}D . \\
\text { palmatum; } \\
D . \\
\text { ruyschiana }\end{array}$ & $\begin{array}{l}\text { Caffeic acid-O-hexoside } \\
\text { [Caffeoyl-O-hexoside] }\end{array}$ & $\mathrm{C}_{15} \mathrm{H}_{18} \mathrm{O}_{9}$ & 342.298 & 341 & & $\begin{array}{l}178 \\
113\end{array}$ & & & {$[27 ; 31 ; 36]$} \\
\hline 30 & $\begin{array}{l}D . \\
\text { ruyschiana }\end{array}$ & Apigenin 7-sulfate & $\mathrm{C}_{15} \mathrm{H}_{10} \mathrm{O}_{8} \mathrm{~S}$ & 350.3001 & 349 & & 269 & 223 & & {$[10 ; 33]$} \\
\hline 31 & $\begin{array}{c}D . \\
\text { palmatum }\end{array}$ & Hinokinin & $\mathrm{C}_{20} \mathrm{H}_{18} \mathrm{O}_{6}$ & 354.3533 & & 355 & $\begin{array}{l}337 \\
189\end{array}$ & $\begin{array}{l}319 \\
226\end{array}$ & & {$[5 ; 26]$} \\
\hline 32 & $\begin{array}{c}D . \\
\text { palmatum }\end{array}$ & Prolithospermic acid & $\mathrm{C}_{18} \mathrm{H}_{14} \mathrm{O}_{8}$ & 358.2990 & & 359 & $\begin{array}{l}341 ; \\
207\end{array}$ & $\begin{array}{r}314 ; \\
267 ; \\
149\end{array}$ & & {$[11 ; 41]$} \\
\hline 33 & $\begin{array}{c}D . \\
\text { palmatum }\end{array}$ & Rosmarinic acid & $\mathrm{C}_{18} \mathrm{H}_{16} \mathrm{O}_{8}$ & 360.3148 & 359 & & 161 & 133 & & {$[11 ; 18 ; 41]$} \\
\hline 34 & $\begin{array}{c}D . \\
\text { ruyschiana }\end{array}$ & Caffeic acid derivative & $\mathrm{C}_{16} \mathrm{H}_{18} \mathrm{O}_{9} \mathrm{Na}$ & 377.2985 & 377 & & $\begin{array}{l}341 ; \\
215\end{array}$ & 179 & & [6] \\
\hline 35 & $\begin{array}{c}D . \\
\text { palmatum }\end{array}$ & Salvianic acid C & $\mathrm{C}_{18} \mathrm{H}_{18} \mathrm{O}_{9}$ & 378.3301 & 377 & & $\begin{array}{l}359 ; \\
315 \\
\end{array}$ & 289 & 229 & [11] \\
\hline 36 & $\begin{array}{c}D . \\
\text { palmatum }\end{array}$ & $\begin{array}{c}\text { Dimethyl- } \\
\text { secoisolariciresinol }\end{array}$ & $\mathrm{C}_{22} \mathrm{H}_{30} \mathrm{O}_{6}$ & 390.4700 & & 391 & $\begin{array}{l}373 ; \\
249 ; \\
121\end{array}$ & $\begin{array}{l}355 \\
225\end{array}$ & $313 ; 226$ & [5] \\
\hline 37 & $\begin{array}{c}D . \\
\text { palmatum }\end{array}$ & Caffeic acid derivative & & 408 & & 409 & $\begin{array}{l}365 ; \\
241\end{array}$ & 241 & & [27] \\
\hline 38 & $\begin{array}{c}D . \\
\text { palmatum }\end{array}$ & Podophyllotoxin & $\mathrm{C}_{22} \mathrm{H}_{22} \mathrm{O}_{8}$ & 414.4053 & & 415 & $\begin{array}{l}248 \\
331\end{array}$ & 145 & & [5] \\
\hline 39 & $\begin{array}{c}D \text {. } \\
\text { ruyschiana }\end{array}$ & Chrysin 6-C-glucoside & $\mathrm{C}_{21} \mathrm{H}_{20} \mathrm{O}_{9}$ & 416.3781 & & 417 & $51 ; 127$ & $\begin{array}{l}333 ; \\
267\end{array}$ & 165 & [19] \\
\hline 40 & $\begin{array}{c}D . \\
\text { ruyschiana }\end{array}$ & $\begin{array}{l}\text { Apocarotenal [(all-E)- } \\
\text { beta-apo-caroten-8'-al] }\end{array}$ & $\mathrm{C}_{30} \mathrm{H}_{40} \mathrm{O}$ & 416.6380 & & 417 & $\begin{array}{l}399 ; \\
200\end{array}$ & 351 & 267 & [14] \\
\hline 41 & $\begin{array}{c}\text { D. } \\
\text { ruyschiana }\end{array}$ & Chrysin glucuronide & $\mathrm{C}_{21} \mathrm{H}_{18} \mathrm{O}_{10}$ & 430.3616 & & 431 & 255 & $\begin{array}{l}255 \\
153 \\
\end{array}$ & 171 & {$[10]$} \\
\hline 42 & $\begin{array}{c}D . \\
\text { palmatum; } \\
D . \\
\text { ruyschiana }\end{array}$ & $\begin{array}{l}\text { Pelargonidin-3-O- } \\
\text { glucoside (callistephin) }\end{array}$ & $\mathrm{C}_{21} \mathrm{H}_{21} \mathrm{O}_{10}$ & 433.3854 & & 433 & 271 & $\begin{array}{l}153 \\
225\end{array}$ & 171 & {$[3 ; 30]$} \\
\hline 43 & $\begin{array}{c}D . \\
\text { palmatum }\end{array}$ & $\begin{array}{l}\text { Prunin [Naringenin-7-O- } \\
\text { glucoside] }\end{array}$ & $\mathrm{C}_{21} \mathrm{H}_{22} \mathrm{O}_{10}$ & 434.3934 & 433 & & $\begin{array}{c}271 ; \\
151\end{array}$ & $\begin{array}{c}269 ; \\
151\end{array}$ & & {$[9 ; 18]$} \\
\hline 44 & $\begin{array}{c}\text { D. } \\
\text { palmatum }\end{array}$ & $\begin{array}{l}\text { Luteolin 7-O-glucoside } \\
\text { [Cynaroside; Luteoloside] }\end{array}$ & $\mathrm{C}_{21} \mathrm{H}_{20} \mathrm{O}_{11}$ & 448.3769 & & 449 & $\begin{array}{l}287 \\
199\end{array}$ & 153 & & $\begin{array}{c}{[2 ; 9 ; 19 ;} \\
31]\end{array}$ \\
\hline 45 & $\begin{array}{c}D . \\
\text { palmatum }\end{array}$ & $\begin{array}{l}\text { Astragalin [Kaempferol } \\
\text { 3-O-glucoside] }\end{array}$ & $\mathrm{C}_{21} \mathrm{H}_{20} \mathrm{O}_{11}$ & 448.3769 & 447 & & $\begin{array}{l}285 ; \\
327\end{array}$ & 241 & 199 & $\begin{array}{c}{[2 ; 9 ; 24} \\
31 ; 38]\end{array}$ \\
\hline 46 & $\begin{array}{c}D . \\
\text { ruyschiana }\end{array}$ & $\begin{array}{c}\text { Apigenin 7-O- } \\
\text { glucuronide }\end{array}$ & $\mathrm{C}_{21} \mathrm{H}_{18} \mathrm{O}_{11}$ & 446.361 & & 447 & 271 & 153 & $271 ; 171$ & {$[6 ; 31]$} \\
\hline
\end{tabular}




\begin{tabular}{|c|c|c|c|c|c|c|c|c|c|c|}
\hline 47 & $\begin{array}{c}D . \\
\text { palmatum; } \\
D \\
\text { ruyschiana }\end{array}$ & Eriodictyol-O-hexoside & $\mathrm{C}_{21} \mathrm{H}_{22} \mathrm{O}_{11}$ & 450.3928 & 449 & & $\begin{array}{l}285 \\
151\end{array}$ & $\begin{array}{l}243 ; \\
151\end{array}$ & & {$[1 ; 10]$} \\
\hline 48 & $\begin{array}{c}D . \\
\text { ruyschiana }\end{array}$ & $\begin{array}{l}\text { Acacetin 7-O-beta-D- } \\
\text { glucuronide }\end{array}$ & $\mathrm{C}_{22} \mathrm{H}_{20} \mathrm{O}_{11}$ & 460.3876 & 459 & & $\begin{array}{l}283 ; \\
343 ; \\
175 \\
\end{array}$ & 268 & 267 & [16] \\
\hline 49 & $\begin{array}{l}D . \\
\text { ruyschiana }\end{array}$ & $\begin{array}{l}\text { Luteolin-7-O-beta- } \\
\text { glucuronide }\end{array}$ & $\mathrm{C}_{21} \mathrm{H}_{18} \mathrm{O}_{12}$ & 462.3604 & & 463 & 287 & 268 & $245 ; 119$ & {$[35 ; 41]$} \\
\hline 50 & $\begin{array}{c}D . \\
\text { ruyschiana }\end{array}$ & $\begin{array}{l}\text { Kaempferol-3-O- } \\
\text { glucuronide }\end{array}$ & $\mathrm{C}_{21} \mathrm{H}_{18} \mathrm{O}_{12}$ & 462.3604 & & 463 & 287 & $\begin{array}{l}268 ; \\
169\end{array}$ & $241 ; 119$ & $\begin{array}{c}{[1 ; 9 ; 10 ;} \\
27]\end{array}$ \\
\hline 51 & $\begin{array}{l}D . \\
\text { ruyschiana }\end{array}$ & $\begin{array}{l}\text { Diosmetin-7-O-beta- } \\
\text { glucoside }\end{array}$ & $\mathrm{C}_{22} \mathrm{H}_{22} \mathrm{O}_{11}$ & 462.4035 & & 463 & 287 & 168 & 123 & {$[16 ; 20]$} \\
\hline 52 & $\begin{array}{c}D . \\
\text { palmatum }\end{array}$ & Peonidin-3-O-glucoside & $\mathrm{C}_{22} \mathrm{H}_{23} \mathrm{O}_{11+}$ & 463.4114 & & 463 & 301 & 286 & $258 ; 140$ & {$[9 ; 30]$} \\
\hline 53 & $\begin{array}{c}D \\
\text { palmatum } \\
\end{array}$ & Cyanidin 3-(acetyl)hexose & $\mathrm{C}_{23} \mathrm{H}_{23} \mathrm{O}_{12+}$ & 491.4215 & & 491 & 287 & $\begin{array}{r}245 ; \\
153 \\
\end{array}$ & 171 & {$[37]$} \\
\hline 54 & $\begin{array}{c}D \\
\text { Duyschiana } \\
\text { re }\end{array}$ & $\begin{array}{c}\text { 3,4-O-dicaffeoylquinic } \\
\text { acid [Isochlorogenic acid } \\
\text { B] }\end{array}$ & $\mathrm{C}_{25} \mathrm{H}_{24} \mathrm{O}_{12}$ & 516.4509 & & 517 & 397 & $\begin{array}{l}337 ; \\
135\end{array}$ & & {$[2 ; 31]$} \\
\hline 55 & $\begin{array}{c}D . \\
\text { palmatum } \\
\end{array}$ & $\begin{array}{l}\text { 2'-Hydroxygenistein O- } \\
\text { glucoside malonylated }\end{array}$ & $\mathrm{C}_{24} \mathrm{H}_{22} \mathrm{O}_{14}$ & 534.4231 & 533 & & 489 & $\begin{array}{l}285 ; \\
326 \\
\end{array}$ & 284 & [38] \\
\hline 56 & $\begin{array}{c}D . \\
\text { palmatum }\end{array}$ & $\begin{array}{l}\text { Luteolin 7-O-beta-D-(6"- } \\
\text { O-malonyl)-glucoside }\end{array}$ & $\mathrm{C}_{24} \mathrm{H}_{22} \mathrm{O}_{14}$ & 534.4231 & & 535 & $\begin{array}{l}436 ; \\
354 ; \\
287 ; \\
214\end{array}$ & $\begin{array}{l}328 ; \\
238\end{array}$ & & {$[7 ; 16]$} \\
\hline 57 & $\begin{array}{c}D . \\
\text { palmatum } \\
\text {. }\end{array}$ & $\begin{array}{l}\text { Acacetin C-glucoside } \\
\text { methylmalonylated }\end{array}$ & $\mathrm{C}_{26} \mathrm{H}_{26} \mathrm{O}_{13}$ & 546.4758 & & 547 & $\begin{array}{l}529 ; \\
496 ; \\
369\end{array}$ & 343 & & [38] \\
\hline 58 & $\begin{array}{c}D . \\
\text { palmatum }\end{array}$ & 5,8-epoxy-alpha-carotene & $\mathrm{C}_{40} \mathrm{H}_{56} \mathrm{O}$ & 552.872 & & 553 & $\begin{array}{l}536 ; \\
412 ;\end{array}$ & $\begin{array}{l}299 ; \\
261\end{array}$ & & {$[8]$} \\
\hline 59 & $\begin{array}{c}D \\
\text { ruyschiana }\end{array}$ & $\begin{array}{l}\text { Apigenin 8-C-pentoside- } \\
\text { 6-C-hexoside }\end{array}$ & $\mathrm{C}_{26} \mathrm{H}_{28} \mathrm{O}_{14}$ & 564.4921 & & 565 & $\begin{array}{l}547 ; \\
274\end{array}$ & $\begin{array}{l}529 ; \\
474 ; \\
247\end{array}$ & 390 & [3] \\
\hline 60 & $\begin{array}{c}D . \\
\text { palmatum }\end{array}$ & $\begin{array}{l}\text { Kaempferol 3-O- } \\
\text { rutinoside }\end{array}$ & $\mathrm{C}_{27} \mathrm{H}_{30} \mathrm{O}_{15}$ & 594.5181 & 593 & & 285 & $\begin{array}{l}241 ; \\
199\end{array}$ & 199 & $\begin{array}{l}{[1 ; 2 ; 9 ; 24 ;} \\
27 ; 31]\end{array}$ \\
\hline 61 & $\begin{array}{l}D . \\
\text { ruyschiana }\end{array}$ & $\begin{array}{l}\text { Violaxanthin [Zeaxanthin } \\
\text { Dieperoxide] }\end{array}$ & $\mathrm{C}_{40} \mathrm{H}_{56} \mathrm{O}_{4}$ & 600.8702 & & 601 & $\begin{array}{l}364 ; \\
582\end{array}$ & $\begin{array}{l}346 ; \\
202 ; \\
142\end{array}$ & 114 & [17] \\
\hline
\end{tabular}

\section{Discussion}

There were identified 61 target analytes in extracts of D. palmatum Stephan and D. ruyschiana L. In the present study, 47 polyphenol compounds were identified and characterized including 14 flavones (Apigenidin, apigenin, luteolin, apigenin 7-sulfate, chrysin 6-C-glucoside, chrysin glucuronide, apigenin 7-O-glucuronide, luteolin 7-Oglucoside, Acacetin 7-O- $\beta$-D-glucuronide, Apigenin 8-C-pentoside-6-C-hexoside, 5 flavonols (kaempferol, dihydrokaempferol, astragalin, Kaempferol-3-O-glucuronide, Kaempferol 3-O-rutinoside, 2 flavanones (Prunin, Eriodictyol- $O$-hexoside), 1 isoflavone (2'-Hydroxygenistein $O$-glucoside malonylated), 1 flavan-3-ol ((epi)catechin), 4 anthocyanidins (Petunidin, Pelargonidin-3-O-glucoside (callistephin), Peonidin-3-Oglucoside, Cyanidin 3-(acetyl)hexose), 3 lignans (hinokinin, Dimethyl-secoisolariciresinol, Podophyllotoxin), 2 stilbenes and 15 phenolic acids.

Figures 3-4 shows examples of the decoding spectra (collision-induced dissociation (CID) spectrum) of the ion chromatogram obtained using tandem mass spectrometry. The $[\mathrm{M}+\mathrm{H}]^{+}$ion produced three fragment ions at $\mathrm{m} / \mathrm{z} 373, \mathrm{~m} / \mathrm{z} 248$, and $\mathrm{m} / \mathrm{z} 121$ (Fig. 3). The fragment ion with $\mathrm{m} / \mathrm{z} 373$ yields two daughter ions at $\mathrm{m} / \mathrm{z} 355$, and $\mathrm{m} / \mathrm{z} 255$. The fragment ion with $\mathrm{m} / \mathrm{z} 355$ yields two daughter ions at $\mathrm{m} / \mathrm{z} 313$, and $\mathrm{m} / \mathrm{z} 226$. This compound was identified in the bibliography as Dimethyl-secoisolariciresinol in extracts from lignans [5]. 


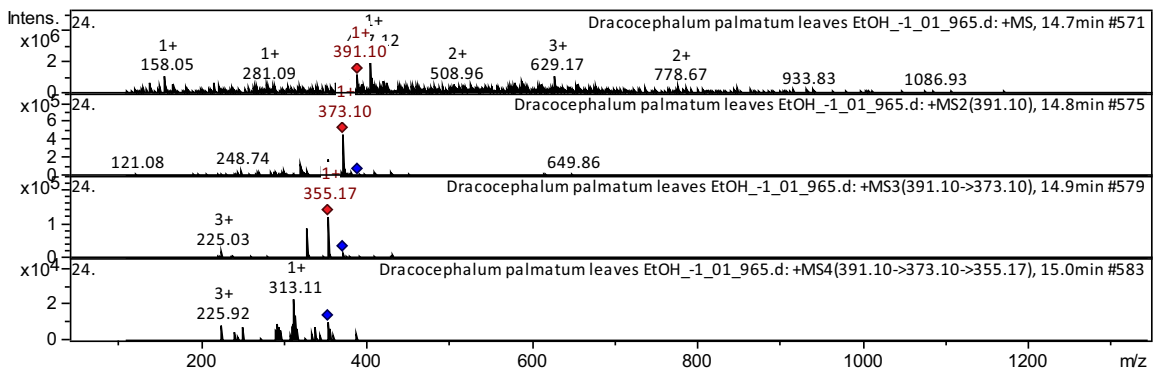

Fig. 3. Mass spectrum of dimethyl-secoisolariciresinol from extracts of D. palmatum, $m / z$ 391.10.

The mass spectrum in positive ion mode of L-tryptophan from extracts of $D$. ruyschiana is shown in Figure 4. The $[\mathrm{M}+\mathrm{H}]+$ ion produced one fragment ions at $m / z 188.02$ (Fig. 4). The fragment ion with $m / z 188.02$ yields one daughter ion at $m / z 144.05$. The fragment ion with $\mathrm{m} / z 144.05$ yields one daughter ion at $\mathrm{m} / z$ 118.08. To our knowledge, L-tryptophan was reported in Passiflora incarnata [19]; Vigna unguiculata [23]; Camellia kucha [24].

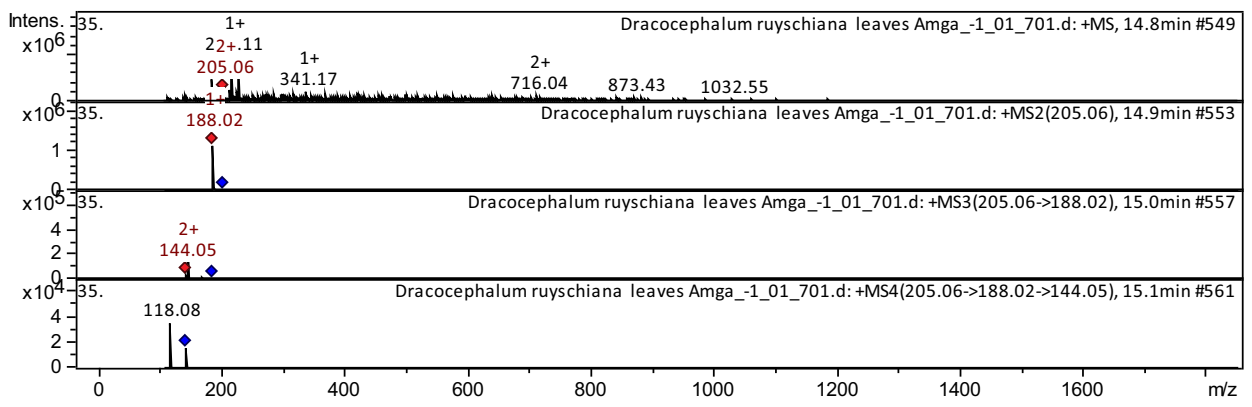

Fig. 4. Mass spectrum of L-tryptophan from extracts of D. ruyschiana, $m / z 205$.

Thus, 61 metabolome compounds were identified in the maceration extracts of $D$. palmatum Stephan and D. ruyschiana L., many of which are characteristic of genus Dracocephalum. Of these, 53 components were identified for the first time in this plant species. These are flavones: Apigenin 8-C-pentoside-6- $C$-hexoside, Apigenin 7-sulfate; Chrysin 6-C-glucoside, Chrysin glucuronide; flavanols: Kaempferol, Dihydrokaempferol, Astragalin; flavan-3-ol (epi)Catechin, phenolic acids: Methylgallic acid; Hydroxy methoxy dimethylbenzoic acid; Ellagic acid; Caffeoylshikimic acid; Prolithospermic acid; 3,4-Odicaffeoylquinic acid; salvianolic acid G; stilbenes pinosylvin and resveratrol; anthocyanins petunidin, Pelargonidin-3-O-glucoside; Peonidin-3-O-glucoside; Cyanidin 3(acetyl)hexose; perillic acid; lignans: Hinokinin, Dimethyl-secoisolariciresinol, Podophyllotoxin, carotenoids: Apocarotenal, 5,8-epoxy-alpha-carotene; etc.

\section{Conclusion}

The extracts of D. palmatum Stephan and D. ruyschiana L. contains a large number of polyphenolic complexes, which are biologically active compounds. For the most complete and safe extraction, the method of maceration with $\mathrm{MeOH}$ was used. To identify target analytes in supercritical extracts, HPLC was used in combination with a BRUKER DALTONIKS ion trap. The results of a preliminary study showed the presence of 61 compounds corresponding to the genus Dracocephalum, of which 53 were identified for the first time in Dracocephalum. 
The data obtained will help to intensify future research on the development and production of various medical products containing targeted extracts of D. palmatum Stephan and D. ruyschiana L. A wide variety of biologically active polyphenolic compounds opens up rich opportunities for the creation of new drugs, as well as biologically active additives based on extracts from the genus Dracocephalum.

Research work according to Project of NEFU "Cell and molecular genetic technologies of research of northern and arctic plants of Yakutia and development on their basis" (SRP № 6, 30.10.2020) and № 0662-2019-0003 "Genetic resources of vegetable and melons of the world collection of N.I. Vavilov All-Russian Institute of Plant Genetic Resources: effective ways of expanding diversity, disclosing the patterns of hereditary variability, use of adaptive potential".

\section{References}

1. I.M. Abu-Reidah, M. S. Ali-Shtayeh, R. M. Jamous, D. Arraes-Roman, A. SeguraCarretero, Food Chem., 166, 179 (2015)

2. Z. Cai, C. Wang, L. Zou, X. Liu, J. Chen, M. Tan, Y. Mei, L. Wei, Molecules, 24, 1936 (2019)

3. G. Dinelli, A. Segura-Carretero, R. Di Silvestro, I. Marotti, D. Arraez-Roman, S. Benedettelli, L. Ghiselli, A. Fernandez-Gutierrez, J. Chromatogr. A., 1218 (2011)

4. D. Ekeberg, P.-O. Flate, M. Eikenes, M. Fongen, C.F. Naess-Andresen, J. of Chromatogr. A, 267 (2006)

5. P.C. Eklund, M. J. Backman, L.A. Kronberg, A.I. Smeds, R.E. Sjoholm, J. of Mass Spectr., 43, 97 (2008)

6. El-Sayed, M.A. Abbas, F.A. Refaat, S. El-Shafae, A.M. Fikry, E. Egyptian J. of Chem., 22, 2 (2021)

7. K.H. Enerstvedt, M. Jordheim, O.M. Andersen, American J. of Plant Science, 7 (2016)

8. L. Etzbach, A. Pfeiffer, F. Weber, A. Schieber, Food Chem., 245 (2018)

9. P. Goufo R.K. Singh, I. A. Cortez, Antioxidants, 9 (2020)

10. A.R. Hamed, S.S. El-Hawary, R.M. Ibrahim, U.R. Abdelmohsen, A.M. El-Halawany, J. Chrom. Sci., 1 (2020)

11. R.-W. Jiang, K.-M. Lau, P.-M. Hon, T.C.W. Mak, K.-S. Woo, K.-P. Fung, Current Med. Chem., 12, 237 (2005)

12. J. Kim, J.N. Kim, I. Park, S. Sivtseva, Z. Okhlopkova, I.S. Zulfugarov, S.-W. Kim, Oncology Reports, 44 (2020)

13. S. Kim, S. Oh, H.B. Noh, S. Ji, S.H. Lee, J.M. Koo, C.W. Choi, H.P. Jhun, Molecules, 23 (2018)

14. S. Lara-Abia, G. Lobo-Rodrigo, J. Welti-Chanes, M. Pilar Cano, Roots. Foods, 10 (2021)

15. S.-E. Lee, Z. Okhlopkova, C. Lim, S. Cho, Chinese J. of Nat. Med., 18, 10 (2020)

16. M. Martinez-Vazquez, R. Estrada-Reyes, A. Martinez-Laurrabaquio, C. LopezRubalcava, G. Heinze, J. of Ethnopharmacol, 141 (2012)

17. A.Z. Mercadante, D.B. Rodrigues, F.C. Petry, L.R. Barros Mariutti, Food Research International, 99, 2 (2017)

18. D.O. Olennikov, N.K. Chirikova, Z.M. Okhlopkova, Molecules, 18 (2013) 
19. M. Ozarowski, A. Piasecka, A. Paszel-Jaworska, D. Siqueira de A. Chaves, A. Romaniuk, M. Rybczynska, A. Gryszczynska, A. Sawikowska, P. Kachlicki, P.L. Mikolajczak, A. Seremak-Mrozikiewicz, Braz. J. Pharmacol, 28 (2018)

20. B.P. Pandey, S.P. Pradhan, K. Adhikari, Chemistry \& Biodiversity, 17, 6 (2020)

21. S. Patnala, I. Kanfer, J. Pharm. Pharm. Sci., 18, 4 (2015)

22. L. Paudel, F. J. Wyzgoski, J. C. Scheerens, A. M. Chanon, R. N. Reese, D. Smiljanic, C. Wesdemiotis, J. J. Blakeslee, K. M. Riedl, P. L. Rinaldi, J. Agr. Food Chem., 61, 49 (2013)

23. I. Perchuk, T. Shelenga, M. Gurkina, E. Miroshnichenko, M. Burlyaeva, Molecules, 25 (2020)

24. D. Qin, Q. Wang, H. Li, X. Jiang, K. Fang, Q. Wang, B. Li, C. Pan, H. Wu, Food Research International, 138 (2020)

25. S.A.O. Santos, C.S.R. Freire, M.R.M. Domingues, A.J.D. Silvestre, C.P. Neto, Food Chem., 59 (2011)

26. M. Sharma, R. Sandhir, A. Singh, P. Kumar, A. Mishra, S. Jachak, S.P. Singh, J. Singh, J. Roy, Front. Plant. Sci., 7 (2016)

27. V. Spinola, J. Pinto, P.C. Castilho, Food Chem., 173, 14 (2015)

28. F. Simard, J. Legault, S. Lavoie, V. Mshvildadze, A. Pichette, Phytother. Res., 22, 919 (2008)

29. State Pharmacopoeia of the Russian Federation, $13^{\text {th }}$ edition (2018)

30. J. Sun, X. Liu, T. Yang, J. Slovin, P. Chen, Food Chem., 146, 289 (2014)

31. L. Sun, S. Tao, S. Zhang, Molecules, 24, 159 (2019)

32. M. Talari, E. Seydi, A. Salimi, et al., Biomed Res. Int. (2014)

33. Y.C.E. Teles, C.C. Rebello Horta, M. de Fatima Agra, W. Siheri, M. Boyd, J. O. Igoli, A.I. Gray, M. de Fatima Vanderlei de Souza, Molecules, 20 (2015)

34. M.C. Thomas, S.R. Dunn, J. S. Altvater, G. Dove, G.W. Nette, Anal. Chem., 84 (2012)

35. N. E. Thomford, K. Dzobo, D. Chopera, A. Wonkam, A. Maroyi, D. Blackhurst, C. Dandara, Molecules, 21, 7 (2016)

36. L. Van Hoyweghen, K. De Bosscher, G. Haegeman, D. Deforce, A. Heyerick, Phytotherapy Res. (2013)

37. V. Vera de Rosso, S. Hillebrand, E. Cuevas Montilla, F.O. Bobbio, P. Winterhalter, A.Z. Mercadante, J. of Food Comp. and Analys, 21 (2008)

38. A. Wojakowska, A. Piasecka, P.M. Garcia-Lopez, F. Zamora-Natera, P. Krajewski, L. Marczak, P. Kachlicki, M. Stobiecki, Phytochem., 92, 71 (2013)

39. J. Xiao, T. Wang, P. Li, R. Liu, Q. Li, K. Bi, Journal of Chromatography, 1028, 33 (2016)

40. J. Xie, C. Ding, Q. Ge, Z. Zhou, X. Zhi, J. of Chromatogr. B., 864, 87 (2008)

41. L. L. Xu, J. J. Xu, K. R. Zhong, Z. P. Shang, F. Wang, R. F. Wang, B. Liu, Molecules, 22 (2017)

42. S. T. Yang, X. Wu, W. Rui, J. Guo, Y. F. Feng, Acta Chromatographica, 27 (2015)

43. Z.-W. Zhu, J. Li, X.-M. Gao, E. Amponsem, L.-Y. Kang, L.-M. Hu, B.-L Zhang. Y.-X. Chang, J. of pharmaceut. and biomedical analys, 62, 162 (2012) 